\title{
VALIDAÇÃO DOS DADOS DE PRECIPITAÇÃO ESTIMADOS PELO CHIRPS PARA O BRASIL
}

\author{
COSTA, Julio - julioczcta@gmail.com \\ Universidade Federal de São João del-Rei / UFSJ \\ PEREIRA, Gabriel - pereira@ufsj.edu.br \\ Universidade Federal de São João del-Rei / UFS]
}
SIQUEIRA, Maria Elisa - elisasiq@usp.br
Universidade de São Paulo / USP
CARDOZO, Francielle - franciellecardozo@ufsj.edu.br Universidade Federal de São João del-Rei / UFS]
SILVA, Viviane Valéria da - viviane.silva.ufsj@gmail.com Universidade Federal de São João del-Rei / UFS]

\begin{abstract}
RESUMO: Entre os elementos do sistema climático que influenciam as atividades socioeconômicas, a precipitação apresenta papel fundamental em áreas tropicais. Regiões como Africa e América do Sul apresentam uma escassez considerável de observações desta variável, uma vez que a rede de estações meteorológicas não cobre sistematicamente todo o território. Neste contexto, produtos de múltiplos sensores, algoritmos e modelos climáticos são utilizados cada vez mais para análises dos elementos atmosféricos, do clima e da influência da precipitação nas características ambientais. Assim, há uma demanda crescente de validação de produtos orbitais e de simulações numéricas de forma a dar suporte aos estudos da distribuição espacial e temporal da precipitação. Este estudo tem como objetivo principal analisar os dados mensais de precipitação do produto Climate Hazards Group InfraRed Precipitation with Stations (CHIRPS) e verificar sua similaridade com os dados de estações meteorológicas do conjunto de informações do Centro de Previsão de Tempo e Estudos Climáticos (CPTEC) do Instituto Nacional de Pesquisas Espaciais (INPE) e do Instituto Nacional de Meteorologia (INMET) para o território brasileiro para os anos de 1998 a 2010. Para avaliar o CHIRPS, foram utilizados dados de 183 estações meteorológicas do INMET/CPTEC. Os resultados indicam que todas as regiões políticas do Brasil apresentaram determinação alta entre as informações do INMET/CPTEC e CHIRPS $(95,4$ $\%$ ), ainda, quando consideramos toda a área do Brasil, a determinação média entre os dados é de $97 \%$, significativa a $p<0,05$, teste t-student. Espacialmente, o noroeste do estado do Amazonas e o sudoeste do Pará apresentaram as maiores diferenças entre o conjunto de dados. As estimativas do CHIRPS ajustadas linearmente com os dados do INMET/CPTEC apresentaram uma concordância mais acentuada.
\end{abstract}

Palavras-chave: Precipitação, Brasil, Dados Orbitais, Validação, Estações Meteorológicas.

VALIDATION OF PRECIPITATION DATA CHIRPS ESTIMATED TO BRAZIL

ABSTRACT: Among the climatic system elements that influence socioeconomic activities, precipitation plays a fundamental role in tropical areas. Regions such as Africa and South America present a considerable shortage of this variable observation, since the network of meteorological stations does not systematically cover the entire territory. In this context, products of multiple sensors, algorithms and climate models are increasingly being used to analyze atmospheric elements, the climate and the influence of precipitation on environmental characteristics. Thus, there is a growing demand in the validation of orbital products and numerical simulations that supports the studies of the 
spatial and temporal distribution of precipitation. The main objective of this work is to analyze the monthly precipitation data of the Climate Hazards Group InfraRed Precipitation with Stations (CHIRPS) product and verify its similarity with the weather station data from the Weather Forecast and Climate Studies Center (CPTEC) from the National Institute of Space Research (INPE) and the National Institute of Meteorology (INMET) for the Brazilian territory for the years 1998 to 2011. To evaluate the CHIRPS, data from 183 meteorological stations of INMET/CPTEC were used. The results indicate that all the political regions of Brazil showed a high determination between INMET/CPTEC and CHIRPS data $(95,4 \%)$, and when is considered the whole area of Brazil, the average determination between the data is $97 \%$, significant at $<0.05$, $t$-student test. Spatially, the northwest of the Amazonas state and the southwest of Pará state presented the largest differences between the data set. The CHIRPS estimates were calibrated according to the linear regression and showed a more consistent agreement with the INMET/CPTEC data.

Keywords: Precipitation, Brazil, Orbital Data, Validation, Weather Stations.

\section{INTRODUÇÃO}

Entre os elementos do sistema climático que influenciam as atividades socioeconômicas, a precipitação apresenta papel fundamental (FIRPO, 2012). Desta forma, excesso ou déficit de precipitação, que pode durar dias ou meses, impactam o ciclo hidrológico e os elementos do sistema climático, propiciando a ocorrência de inundações e secas extremas (TWARDOSZ et al., 2015). Os dados climáticos são essenciais para a gestão pública no que se refere ao gerenciamento dos recursos hídricos, podendo, desta forma, proporcionar a avaliação do impacto da precipitação no meio ambiente, incluindo a agricultura (WANDERLEY, 2013).

Assim, para aumentar a confiabilidade dos resultados nas análises temporais dos estudos de precipitação, é necessária boa qualidade dos dados estimados, assim como boa disponibilidade e distribuição espacial (COSTA et al., 2012). Em regiões como África e América do Sul há ainda uma grande escassez de informações de estações meteorológicas, além da falta de acesso aos dados de precipitação. Desta forma, observa-se a existência de diversas lacunas espaciais de amostragem de dados, o que é especialmente evidente em regiões menos desenvolvidas ou com baixa densidade populacional. Recursos como produtos de sensores orbitais, algoritmos interpoladores e modelos atmosféricos são cada vez mais necessários para a representação da precipitação em áreas onde não se dispõe da observação da precipitação, se tornando uma opção para estudos climáticos para regiões tropicais e subtropicais, como as ocupadas pelo território brasileiro (SMITH et al., 2012; BAYISSA et al., 2017).

Como os dados da precipitação obtida dos conjuntos de reanálises não apresentam ainda as melhores estimativas (HARADA et al., 2016), a estimativa da precipitação feita a partir de observações remotas e locais constituem atualmente a opção para se obter dados de precipitação distribuídos globalmente com resolução espacial relativamente alta. O CPC Merged Analysis of Precipitation (CMAP), Climate Research Unit (CRU), Tropical Rainfall Measuring Mission (TRMM) e o Climate Hazards group Infrared Precipitation with Stations (CHIRPS) constituem conjuntos de dados de precipitação obtidos a partir de observações locais e remotas. Novos produtos são originados apresentando melhorias consideráveis na extensão, resolução espacial e resolução temporal, o que permite obter dados para quase todo o globo a cada três horas e com resoluções espaciais de aproximadamente 0,050 (COMPO et al., 2006; SMITH et al., 2012). 
Diversos estudos utilizaram bases de dados provenientes de estações meteorológicas e produtos de satélite para analisar a acurácia das estimativas de precipitação (PEREIRA et al., 2013; CAMPAROTTO et al., 2013b; SOARES et al., 2016; ERAZO et al., 2018). Em geral, os autores citados constatam uma alta determinação entre os diferentes produtos disponíveis e os dados coletados. Porém, devido a diferentes métodos e existência de produtos diversificados que variam para cada região nos quesitos espaciais e temporais, é de suma importância a validação dos dados do CHIRPS, a partir de uma série temporal consolidada que abrange o território brasileiro (PEREIRA et al., 2013). Na literatura é possível encontrar alguns trabalhos que avaliam a acurácia do CHIRPS para algumas regiões do globo como África Ocidental, o estado de Minas Gerais, Moçambique, Chile, Bacia do Adige (Itália), e as pesquisas ressaltam a confiabilidade nos dados do produto (TOTÉ et al., 2015; DEMBÉLÉ et al., 2016; DUAN et al., 2016; ZAMBRANO et al., 2017 NOGUEIRA et al., 2018).

Dentre os países da América do Sul, o Brasil é o que apresenta a maior extensão territorial. Apresenta seis complexos domínios de paisagem, sendo dois subtropicais e quatro intertropicais. Inseridas em uma área de 2 milhões de $\mathrm{km}^{2}$, as florestas da Amazônia, um dos domínios paisagísticos do Brasil, são compostas por uma área de planícies de inundações e drenagens perenes. Os cerrados, florestas galerias e outros ambientes de planaltos caracterizam o domínio Chapadões localizados na porção central do país. Os "mares de morro" caracterizam-se pela presença das florestas atlânticas e pela forte presença de decomposição de rochas cristalinas. No sul do Brasil, o domínio das padrarias apresenta ecologia de zona temperada subúmida. Os planaltos das Araucárias são cobertos por bosques de araucárias em seus planaltos médios. O domínio das depressões interplanálticas semiáridas do Nordeste apresenta área de planícies de erosão (AB'SABER, 1977).

Neste contexto, o presente trabalho tem como objetivo principal validar os dados de precipitação estimados pelo CHIRPS em relação aos dados observados em estações meteorológicas do conjunto de informações do Centro de Previsão de Tempo e Estudos Climáticos (CPTEC) do Instituto Nacional de Pesquisas Espaciais (INPE) e do Instituto Nacional de Meteorologia (INMET), distribuídas pelo território brasileiro, para os anos de 1998 a 2010.

\section{DADOS E MÉTODOS}

\subsection{CHIRPS}

O Climate Hazards Group InfraRed Precipitation with Stations (CHIRPS) é um conjunto de dados de precipitação desenvolvido pelo United States Geological Survey (USGS) e pelo Climate Hazards Group at the University of California, Santa Barbara (UCSB). Nesse produto, as estimativas de precipitação são compostas por diversas fontes de informações, como: (I) The Climate Hazards Group's Precipitation Climatology (CHPClim); (II) Observações de satélites com espectroscopia de infravermelho termal (Thermal Infrared, TIR), geoestacionárias quase globais da National Oceanic and Atmospheric Administration (NOAA), Centro de Previsão Climática (CPC) e o National Climatic Data Center Climáticos (NCDC); (III) Campos de Precipitação do Coupled Forecast System da NOAA, versão 2 (CFSv2); (IV) Diversas observações de precipitação através de produtos de estações meteorológicas e outros serviços regionais (FUNK et al., 2015). 
O produto CHIRPS possui uma resolução espacial de $0,05^{\circ}$, ou aproximadamente $5 \mathrm{~km}$, próximo ao equador, cobertura geográfica de $50^{\circ} \mathrm{S}$ a $50^{\circ} \mathrm{N}$, com dados de 1981 até os dias atuais e é disponibilizado em conjuntos de dados diários, em pêntadas e dados mensais. Os dados do CHIRPS foram utilizados neste trabalho para analisar a distribuição espacial da precipitação sobre o Brasil e sua validação foi feita a partir das estações meteorológicas do INMET/CPTEC. Os dados do CHIRPS estão disponíveis no sítio eletrônico da UCSB (ftp://ftp.chg.ucsb.edu/pub/org/chg/products/CHIRPS-2.0/), em formato NetCDF, GeoTiff e Esri BIL.

\subsection{DADOS PLUVIOMÉTRICOS}

Com o objetivo de avaliar os dados estimados pelo produto CHIRPS, foram utilizados dados de estações meteorológicas disponibilizados pelo INMET e CPTEC. Estas informações são obtidas a partir do Surface Synoptic Observations (SYNOP/INMET), das plataformas de coleta de dados do Centro de Missão de Coleta de Dados do Instituto Nacional de Pesquisas Espaciais (CMD/INPE), e também do Programa de Monitoramento de Tempo, Clima e Recursos Hídricos (PMTCRH) (PEREIRA et al., 2013). A localização das estações meteorológicas utilizadas é visualizada na Figura 1.

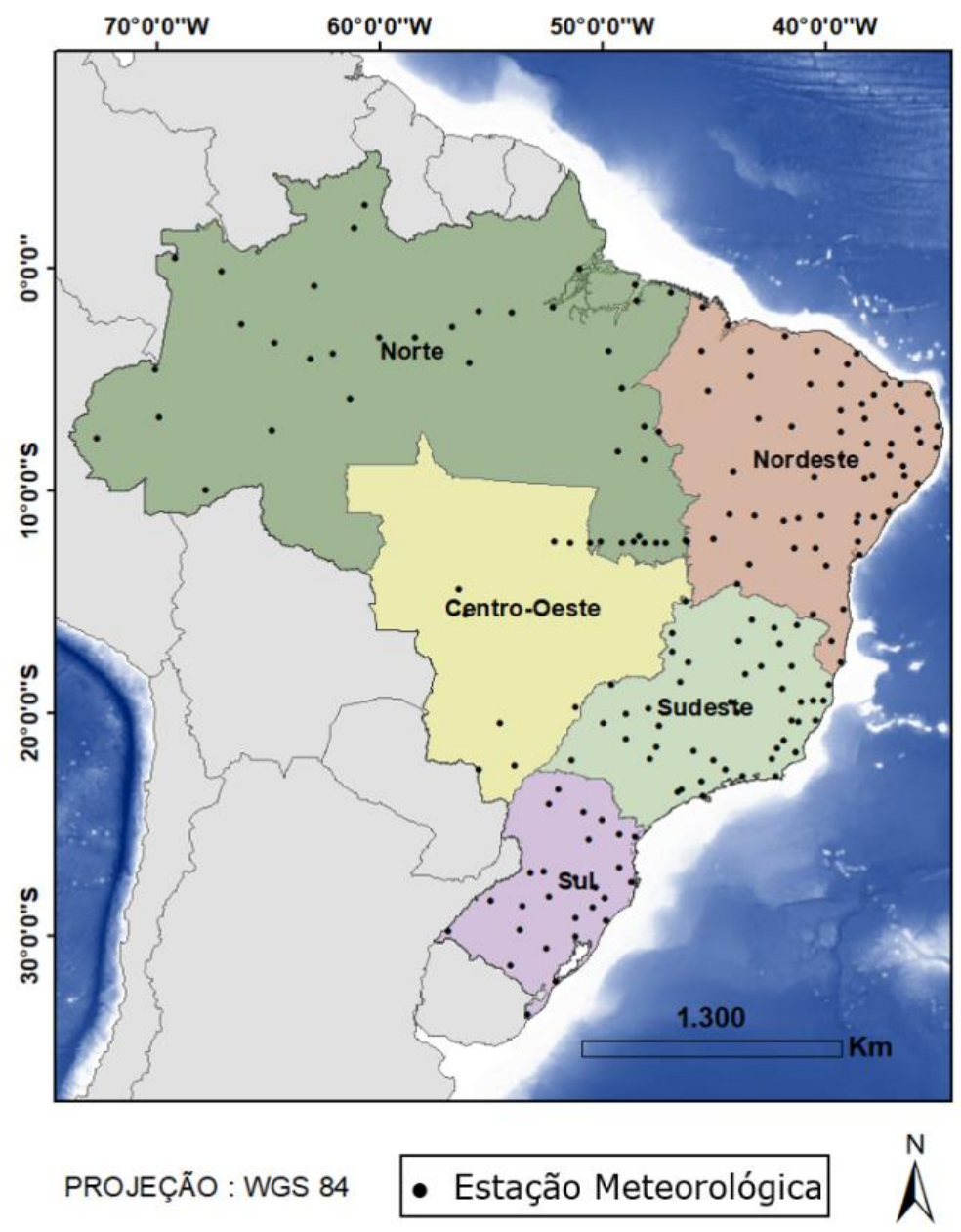


Figura 1 - Localização das estações meteorológicas selecionadas no território brasileiro.

Os dados de precipitação, disponibilizados pelo Grupo de Previsão Climática do INPE, possuem diferentes períodos de abrangência, que variam de acordo com a variável meteorológica e com a frequência temporal (mensal ou diário). Os dados mensais são disponibilizados do ano de 1961 a 2010 e os dados diários a partir de 1997. Porém, apenas a partir do ano de 1998 que obteve dados confiáveis, iniciando tal ano a validação da presente pesquisa. Grande parte das estações meteorológicas do Brasil estão localizadas na faixa litorânea, ou, próximas à rede hidrográfica. No total foram utilizadas 183 estações meteorológicas (Figura 1), das quais 64 estão localizadas na região Nordeste, 39 no Norte, 8 estão se localizam no Centro-Oeste, 27 estão na região Sul e 45 no Sudeste.

\subsection{VALIDAÇÃO DOS DADOS DO CHIRPS}

Os dados do CHIRPS e das estações foram comparados a partir do cálculo do coeficiente de determinação $\left(r^{2}\right)$, do Erro médio quadrático (EMQ), do erro sistemático (BIAS) e do erro absoluto, para a escala mensal. Os cálculos foram feitos para todos os postos selecionados e para as regiões políticas do Brasil.

O coeficiente determinação $\left(r^{2}\right)$ é utilizado para avaliar a associação entre dois fatores, através da proporção da variabilidade em uma variável, e pode ser expresso pela equação 1, segundo BAYISSA et al. (2017):

$$
r=\frac{\sum(\hat{y} i-\bar{y})^{2}}{\sum\left(y_{i}-\bar{y}\right)^{2}}
$$

Na equação 1, $\hat{y} i$ representa o valor estimado, $y i$ representa o valor observado, $\overline{\mathrm{y}}$ corresponde à média das observações, levando a soma total dos quadrados (BAYISSA et al., 2017).

O BIAS ou Viés corresponde à média do erro sistemático em cada tempo, introduzido nas medidas por uma série de fatores. O BIAS representa a média dos erros individualizados, e é geralmente utilizado para saber se há subestimativa ou superestimativa média dos valores estimados. Ressalta-se que erros com sinais opostos contribuem para que o valor médio se anule. Se o BIAS é igual a zero, pode-se afirmar que não há erro sistemático ou tendência no conjunto de dados estimados. Na equação 2, $s$ corresponde aos valores dos dados do CHIRPS, enquanto " $O$ " representa os dados das observados nas estações meteorológicas e $\mathrm{n}$ representa $\mathrm{o}$ tamanho da série temporal considerada (BAYISSA et al., 2017).

$$
\mathrm{BIAS}=\frac{1}{n} \sum_{i=1}^{n}(s-o)
$$

O erro quadrático médio (EQM) foi utilizado com o propósito de avaliar a intensidade média dos erros entre os dados do CHIRPS e de estações meteorológicas. Na equação 3, EQM equivale ao erro quadrático médio, onde o símbolo s corresponde aos valores dos dados do CHIRPS, enquanto o representa os dados das estações meteorológicas (BAYSSA et al., 2017). 


$$
\operatorname{RMSE}=\left[\frac{1}{n} \sum_{i=1}^{n}(s-o)^{2}\right]^{1 / 2}
$$

O erro absoluto médio (E) é uma análise estatística menos afetada pelos outliers. Esta análise representa a soma cumulativa dos erros e apresenta uma estimativa robusta para indicar a habilidade dos dados estimados em reproduzir os valores observados. Na equação 4, o símbolo s corresponde aos valores dos dados do CHIRPS, enquanto o representa os dados das estações meteorológicas (MELLO et al., 2009; CAMPAROTTO et al., 2013a).

$$
\mathrm{E}=\frac{1}{N} \sum_{i=1}^{n}|s-o|
$$

Adicionalmente, os padrões de distribuição espacial da precipitação média para o período considerado, 1998-2010, com dados do CHIRPS e de estações meteorológicas foram comparados. Neste caso, houve a necessidade de interpolação dos dados das estações meteorológicas (figura 2) para a grade do CHIRPS, a partir da interpolação por Krigagem obtida diretamente da fonte dos dados. A Figura 2 esquematiza as etapas até a avaliação final dos dados do CHIRPS.

Nesse contexto, com a finalidade de avaliar as oscilações entre a base de dados de precipitação do CHIRPS e a base de dados do INMET/CPTEC, inicialmente foram isolados índices mensais e posteriormente as médias mensais foram agrupadas por região. Considerando os valores de coeficiente de determinação $\left(r^{2}\right)$, raiz do erro médio quadrático (RMSE), erro absoluto (\%) e erro sistemático (BIAS) para cada região do Brasil, os índices estão representados na Tabela 1 .

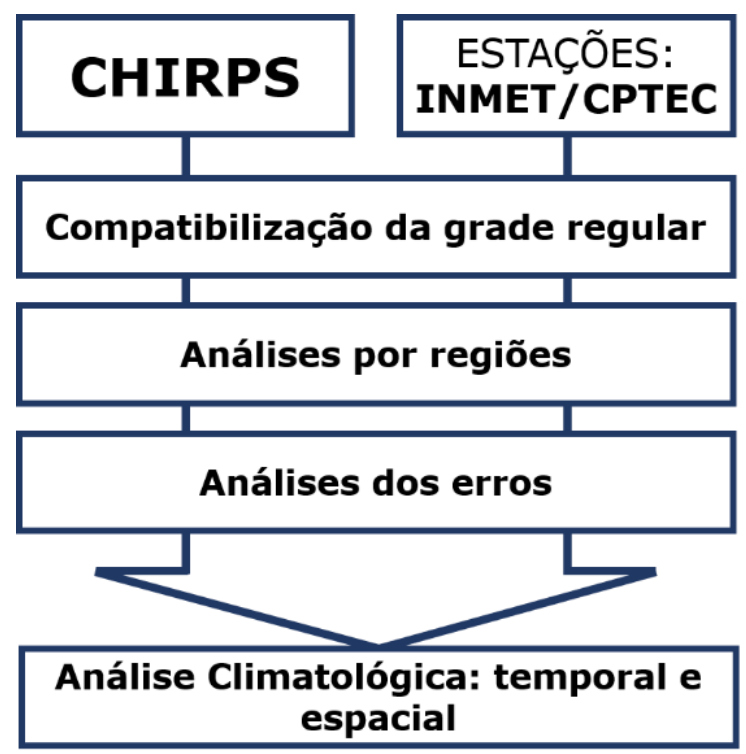

Figura 2 - Fluxograma da estratégia de análise utilizada.

\section{RESULTADOS E DISCUSSÃO}

A Figura 4 apresenta a média mensal da precipitação acumulada para o CHIRPS (em branco) e para as estações meteorológicas do INMET/CPTEC (em azul). Com isso, podemos observar que nas regiões Centro-Oeste e Sudeste a 
sazonalidade da chuva pode ser classificada em 3 fases: estação chuvosa (janeiro - abril), estação seca (maio - setembro) e estação de transição (outubro - dezembro). A região Norte apresenta um período de chuva entre novembro a maio e estiagem entre junho a setembro, caracterizando deste modo a região brasileira com maior período de chuva e índices de precipitação elevados mesmo na estação seca. O Nordeste tem seu período de chuva entre os meses de janeiro e maio e uma estação seca entre julho a dezembro, assim, os baixos índices indicam o Nordeste como a região mais seca do Brasil. Enquanto isso, na região Sul os índices não apresentaram grande variabilidade, ou seja, um período seco definido. Desta forma, na estação chuvosa as regiões Centro-Oeste e Norte se sobressaem com menores coeficientes de determinação, sendo de 78\% (Centro-Oeste) e 77\% (Norte), consideravelmente abaixo das demais regiões, que apresentam em média determinação de $94 \%$ entre os conjuntos de dados. O Centro-Oeste se destacou com uma superestimativa de erro médio em $10 \mathrm{~mm}$ em comparação entre os dados do INMET/CPTEC e do CHIRPS, conforme os dados da Tabela 1.

Tabela 1 - Coeficiente de determinação $\left(r^{2}\right)$, RMSE, BIAS, e Erro Absoluto para cada mês do ano, entre janeiro de 1998 e dezembro de 2010.

\begin{tabular}{|c|c|c|c|c|c|}
\hline Mês & Região & $\begin{array}{c}\text { Coeficiente } \\
\text { de } \\
\text { determinação }\end{array}$ & RMSE & BIAS & $\begin{array}{c}\text { Erro } \\
\text { Absoluto }\end{array}$ \\
\hline \multirow[b]{5}{*}{ Janeiro } & Centro-Oeste & 0.78 & 51.20 & 21.12 & 18.20 \\
\hline & Norte & 0.65 & 32.77 & 11.48 & 7.78 \\
\hline & Nordeste & 0.99 & 13.43 & 11.23 & 7.82 \\
\hline & Sul & 0.87 & 20.05 & 8.43 & 2.73 \\
\hline & Sudeste & 0.98 & 22.84 & 10.18 & 10.18 \\
\hline \multirow[b]{5}{*}{ Fevereiro } & Centro-Oeste & 0.82 & 28.53 & 12.00 & 1.68 \\
\hline & Norte & 0.66 & 30.42 & 10.85 & 4.82 \\
\hline & Nordeste & 0.98 & 10.16 & 7.69 & 1.15 \\
\hline & Sul & 0.95 & 17.41 & 9.19 & -2.35 \\
\hline & Sudeste & 0.97 & 12.85 & 8.76 & 4.67 \\
\hline \multirow{9}{*}{ Março } & Centro-Oeste & 0.77 & 30.65 & 15.81 & 10.05 \\
\hline & Norte & 0.86 & 22.17 & 6.34 & 1.89 \\
\hline & Nordeste & 0.95 & 12.88 & 6.99 & 4.92 \\
\hline & Sul & 0.94 & 12.68 & 7.75 & 2.76 \\
\hline & Sudeste & 0.96 & 11.61 & 6.62 & 3.89 \\
\hline & Centro-Oeste & 0.78 & 19.02 & 16.48 & 7.30 \\
\hline & Norte & 0.91 & 23.13 & 9.29 & 6.71 \\
\hline & Nordeste & 0.99 & 9.74 & 5.92 & 2.86 \\
\hline & Sul & 0.98 & 14.58 & 8.33 & -0.45 \\
\hline \multirow[t]{5}{*}{ Abril } & Sudeste & 0.97 & 8.52 & 14.68 & 11.47 \\
\hline & Centro-Oeste & 0.94 & 10.55 & 17.90 & 16.04 \\
\hline & Norte & 0.91 & 20.11 & 9.43 & 8.65 \\
\hline & Nordeste & 0.99 & 6.34 & 5.91 & 0.70 \\
\hline & Sul & 0.98 & 8.51 & 5.60 & -2.61 \\
\hline \multirow[t]{5}{*}{ Maio } & Sudeste & 0.98 & 2.73 & 19.85 & 13.73 \\
\hline & Centro-Oeste & 0.97 & 6.32 & 48.73 & 37.89 \\
\hline & Norte & 0.73 & 20.58 & 16.60 & 14.46 \\
\hline & Nordeste & 0.78 & 13.39 & 12.57 & -0.97 \\
\hline & Sul & 0.88 & 17.47 & 17.84 & 8.09 \\
\hline Junho & Sudeste & 0.98 & 2.73 & 19.85 & 13.73 \\
\hline
\end{tabular}




\begin{tabular}{|c|c|c|c|c|c|}
\hline \multirow[b]{5}{*}{ Julho } & Centro-Oeste & 0.91 & 7.48 & 45.87 & 9.77 \\
\hline & Norte & 0.56 & 16.68 & 16.37 & 15.54 \\
\hline & Nordeste & 0.89 & 7.53 & 10.86 & 3.33 \\
\hline & Sul & 0.91 & 13.41 & 10.60 & 1.48 \\
\hline & Sudeste & 0.93 & 4.62 & 22.64 & 8.54 \\
\hline \multirow[b]{5}{*}{ Agosto } & Centro-Oeste & 0.96 & 9.27 & 108.44 & 96.57 \\
\hline & Norte & 0.64 & 13.55 & 20.64 & 20.26 \\
\hline & Nordeste & 0.74 & 8.01 & 13.21 & -7.06 \\
\hline & Sul & 0.98 & 12.60 & 12.45 & 7.10 \\
\hline & Sudeste & 0.97 & 4.28 & 42.78 & 32.78 \\
\hline \multirow[b]{5}{*}{ Setembro } & Centro-Oeste & 0.97 & 10.61 & 27.18 & 25.77 \\
\hline & Norte & 0.93 & 9.69 & 12.53 & 11.71 \\
\hline & Nordeste & 0.97 & 6.91 & 15.01 & 3.60 \\
\hline & Sul & 0.99 & 12.73 & 6.76 & 0.24 \\
\hline & Sudeste & 0.98 & 5.73 & 13.39 & 8.50 \\
\hline \multirow[b]{5}{*}{ Outubro } & Centro-Oeste & 0.84 & 26.33 & 21.37 & 18.08 \\
\hline & Norte & 0.71 & 14.64 & 12.11 & 9.75 \\
\hline & Nordeste & 0.97 & 6.25 & 24.05 & 15.83 \\
\hline & Sul & 0.97 & 11.65 & 5.98 & -1.22 \\
\hline & Sudeste & 1.00 & 12.57 & 14.87 & 14.87 \\
\hline \multirow[b]{5}{*}{ Novembro } & Centro-Oeste & 0.84 & 20.37 & 10.57 & 7.77 \\
\hline & Norte & 0.89 & 18.84 & 11.79 & 10.18 \\
\hline & Nordeste & 0.96 & 6.16 & 12.07 & 6.61 \\
\hline & Sul & 0.97 & 15.00 & 8.95 & -1.18 \\
\hline & Sudeste & 0.92 & 19.44 & 8.75 & 8.31 \\
\hline \multirow[b]{5}{*}{ Dezembro } & Centro-Oeste & 0.85 & 35.13 & 15.58 & 15.18 \\
\hline & Norte & 0.89 & 23.50 & 10.92 & 5.84 \\
\hline & Nordeste & 0.97 & 10.88 & 16.14 & 12.07 \\
\hline & Sul & 0.95 & 14.86 & 8.43 & 2.12 \\
\hline & Sudeste & 0.94 & 20.82 & 8.10 & 8.10 \\
\hline
\end{tabular}



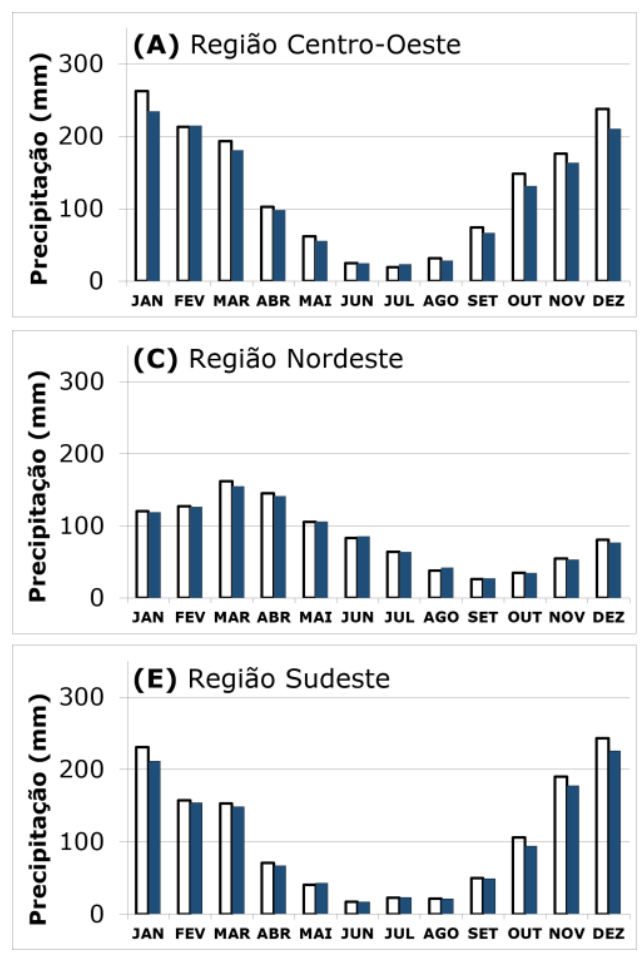
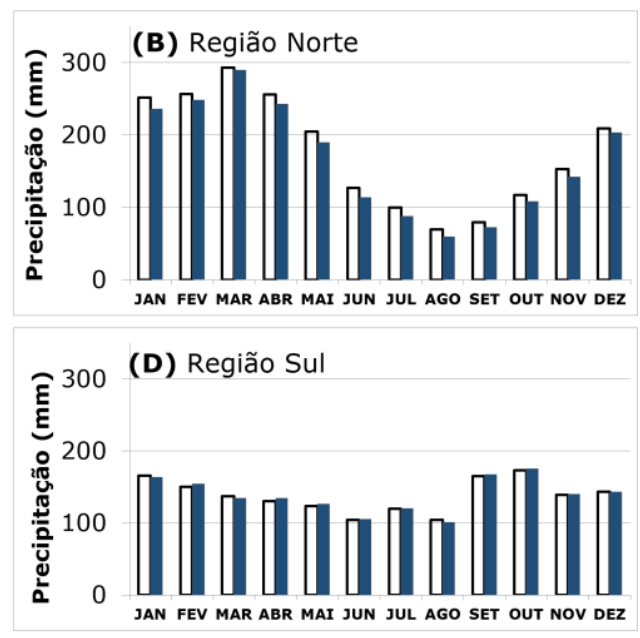

$\square$ CHIRPS

$\square$ CPTEC/INMET

Figura 3 - Média mensal da precipitação para o período de 1998-2010, para as regiões políticas do Brasil: (A) Centro-Oeste, (B) Norte, (C) Nor/deste, (D) Sul e (E) Sudeste.

A comparação entre os dados mensais estimados (CHIRPS) e observados (estações meteorológicas) entre janeiro de 1998 e dezembro de 2010 mostra que o coeficiente de determinação considerando todas as estações meteorológicas selecionadas (Figura 4F), é igual a 95,4 \%, indicando uma forte associação entre os dois conjuntos de dados para o território brasileiro. Todas as regiões políticas brasileiras apresentam coeficientes de determinação superiores a $92 \%$ (Figuras 4A-4E). Os cálculos do coeficiente de determinação entre os dados do CHIRPS e estações meteorológicas foram testados quanto à significância estatística para $\mathrm{p}=0,05$, de acordo com o teste t-Student. 

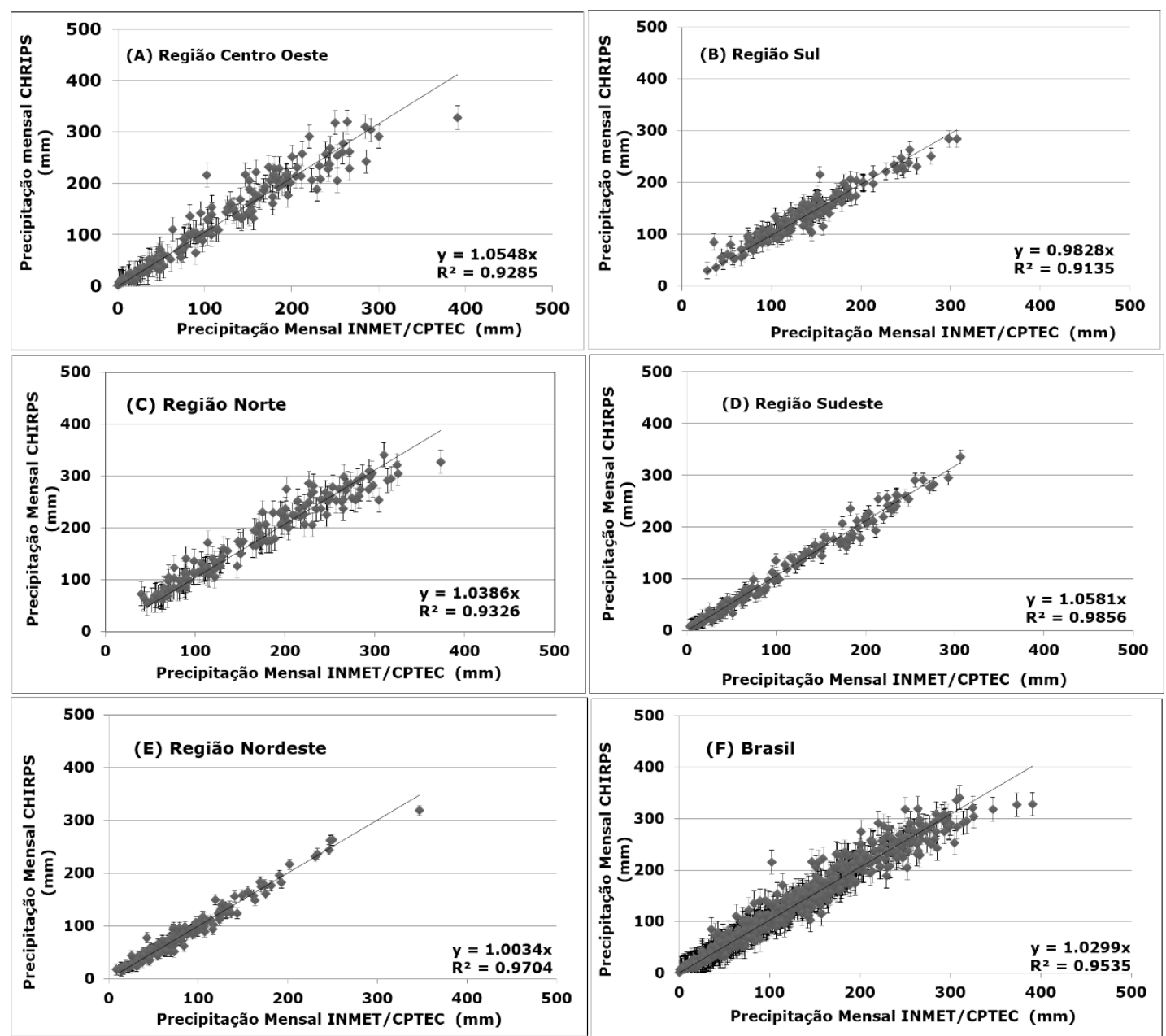

Figura 4 - Coeficiente de determinação entre os dados mensais de precipitação $(\mathrm{mm})$ para janeiro de 1998 a dezembro de 2010 (significante a $p<0,05$, teste t-student), para as regiões políticas do Brasil (A) Centro-Oeste, (B) Sul, (C) Norte, (D) sudeste, (E) Nordeste e para todo o território brasileiro (F) Brasil.

A região centro-oeste, representada por oito estações meteorológicas, apresenta coeficiente de determinação de $96 \%$ (Figura 4A). Os dados do CHIRPS apresentam uma superestimativa de aproximadamente $5 \%$ em relação aos dados das estações meteorológicas. A região Centro-Oeste do Brasil apresenta elevada biodiversidade e compreende a área do Bioma Pantanal, considerada a maior planície alagável do mundo. As circulações atmosféricas que mais afetam o Centro-Oeste apresentam origem tropical e extratropical, sendo influenciadas pelo aquecimento local, pelo transporte de umidade do norte da América do Sul, por sistemas frontais e por massas de ar seco da região subtropical do Atlântico Sul. O relevo é um dos grandes fatores determinantes da variação da temperatura do ar no Centro-Oeste, com áreas de baixas altitudes apresentando valores mensais médios mais elevados de temperatura $\left(\sim 33^{\circ} \mathrm{C}\right)$ e áreas com altas altitudes apresentando temperaturas médias mais baixas $\left(\sim 26^{\circ} \mathrm{C}\right)$ (CAVALCANTE et al., 2009$)$.

A região Sul apresenta 95\% como coeficiente de determinação entre os dados do CHIRPS e os dados das estações meteorológicas e $2 \%$ de subestimativa em relação aos dados observados. $\mathrm{Na}$ região Sul, as 
características de relevo e sua localização em médias latitudes proporcionam variações na sazonalidade dos elementos climáticos. O norte da região Sul caracteriza-se por um clima tipo monções, com aumento dos índices pluviométricos na primavera, ápice no verão e diminuição no início do outono. Enquanto isso, mais ao sul desta região, a distribuição da chuva no ano é mais homogênea devido tanto à influência do aquecimento local durante o verão como à atuação de frentes frias durante o inverno. A região Sul apresenta os menores valores de temperatura do ar no Brasil, variando de acordo com a latitude (devido à distribuição desigual de irradiância) e a altitude. A região da Serra Geral, em Santa Catarina, com altitudes de até 1.800 metros, apresenta os menores valores de temperatura do ar registrados no Brasil, com valores médios de $11^{\circ} \mathrm{C}$ no mês de julho (CAVALCANTE et al., 2009). Ao mesmo tempo, a topografia acentuada da região contribui para o aumento do volume pluviométrico, alcançando valores médio anual entre 1250 a 2000 mm (CAVALCANTE et al., 2009). O aporte de umidade proveniente do Oceano Atlântico associado às baixas temperaturas durante 0 inverno permite a precipitação de neve nas Serras Catarinenses e Gaúchas (CAVALCANTE et al., 2009; CERA et al., 2015).

Para a região norte, (Figura $4 \mathrm{C}$ ) a regressão indica uma superestimativa de $3 \%$ do dado CHIRPS em relação aos dados do INMET/CPTEC, apresentando $97 \%$ de coeficiente de determinação entre a base de dados (significante a $\mathrm{p}<0,05$, teste t-student). A região Norte do Brasil, onde se localiza a bacia Amazônica, é considerada uma região com capacidade de influenciar o clima em escala global (ARAGÃO et al., 2014; SWANN et al., 2015; FEARNSIDE, 2018; MENEZES et al., 2018), podendo a floresta interferir em variáveis climáticas como a circulação atmosférica e a precipitação regional.

Os resultados obtidos para a região Sudeste (Figura 4D) apresentam o maior coeficiente de determinação entre as regiões administrativas brasileiras, valor de $99 \%$ (significante a $p<0,05$, teste t-student) e, assim como a região Centro-Oeste (Figura 4A), apresentou os valores de precipitação superestimados em aproximadamente 5\%. Deste modo, as regiões Centro-Oeste e Sudeste apresentaram as maiores superestimativas em relação aos dados das estações meteorológicas. Tal fato pode estar associado aos sistemas meteorológicos que atuam nas duas regiões durante as estações do ano, geralmente marcadas por uma estação seca e outra chuvosa. Além disso, a região sudeste concentra cerca de $42,6 \%$ da população brasileira, principalmente nas capitais dos estados de São Paulo, Rio de Janeiro e Minas Gerais (CAVALCANTE et al., 2009). Esta região contém alto índice demográfico e abrange diversos setores da economia, como o setor industrial e a agropecuária, afirmando a importância e a confiabilidade dos dados de precipitação do CHIRPS para a região de Minas Gerais, segundo Nogueira et al. (2018). Desta forma, as atividades econômicas e a sua densidade demográfica levam à demanda por abastecimento urbano e geração de energia, tornando a precipitação um elemento das condições naturais de fundamental importância. Localizada próxima ao Trópico de Capricórnio, a região Sudeste apresenta a interferência de vários fenômenos atmosféricos, com destaque para a Zona de Convergência do Atlântico Sul (ZCAS), bem caracterizada no verão (CAVALCANTE et al., 2009; VÁSQUEZ et al., 2018).

A Figura 4E apresenta os resultados obtidos para a região Nordeste. Nesta região verifica-se uma boa concordância entre os dados estimados pelo 
CHIRPS e os dados das estações meteorológicas, que tendem a similaridade. Nesta, há um coeficiente de determinação de $98 \%$ (significante a $p<0,05$, teste t-student). O Nordeste é a região com maior número de estados e caracterizase por três diferentes tipos de clima: Tropical, mais ao sul; litorâneo úmido, compreendido na região litorânea da Bahia até o Rio Grande do Norte; e Tropical Semiárido, representado pelo Sertão Nordestino. Sua posição a leste da Amazônia, onde ocorre forte convecção local e, consequentemente, altos índices de precipitação, é o fator que determina a escassez de precipitação, sendo caracterizada por forte movimento descendente do ar (REBOITA et al., 2010). O relevo é um aspecto coadjuvante que contribui para a distribuição desigual da precipitação na região. Entre os planaltos Borborema, bacia do rio Parnaíba e as chapadas Diamantina e Araripe se localizam regiões de depressões que caracterizam o sertão, área mais árida e com menores índices pluviométricos (CAVALCANTE et al., 2009). Ao analisar a distribuição dos dados do CHIRPS e sua relação para todo o Brasil (Figura 4F) percebe-se que os dados se encontram superestimados em $2 \%$, sendo associados a erros sistemáticos e pelos mecanismos que originam a precipitação.

De modo geral, a média dos dados mensais do CHIRPS e do INMET/CPTEC obteve boa determinação no território brasileiro, cerca de $91 \%$. Além disso, o RMSE indicou valores médios de $15 \mathrm{~mm}$. A média mensal também demonstrou que os valores do CHIRPS superestimam em erro médio, aproximadamente $9 \mathrm{~mm}$ em relação aos dados do INMET/CPTEC. Entretanto, a precipitação não se difere apenas por regiões, mas apresenta variações relacionadas à sazonalidade. Desta forma, nota-se na Figura 3 que ambos os dados apresentam uma estimativa eficiente da variabilidade sazonal da precipitação que ocorre em cada região.

Para este trabalho, os dados de precipitação foram distribuídos espacialmente para as regiões brasileiras para o período de 1998 a 2010, conforme pode ser visualizado na Figura 5. A Figura $5 \mathrm{~A}$ representa a média anual da precipitação para os dados das estações meteorológicas do INMET/CPTEC, a Figura 5B representa os índices médios da precipitação anuais estimados a partir do CHIRPS e a Figura 5C apresenta os dados de precipitação ajustada pela regressão entre os dados das estações e do CHIRPS. Os 13 anos analisados para o CHIRPS apresentaram concordância favorável da distribuição espacial das médias mensais de precipitação. O noroeste do estado do Amazonas se destaca como uma das poucas regiões que demonstraram heterogeneidade, com os dados das estações meteorológicas variando entre 150 e $200 \mathrm{~mm}$, e os dados CHIRPS entre 150 e $250 \mathrm{~mm}$.

Outra variação significativa ocorre ao sudoeste do estado do Pará, os dados das estações variam entre 140 a $180 \mathrm{~mm}$ e os dados do CHIRPS variam entre 180 a $250 \mathrm{~mm}$. Segundo Pereira et al. (2013) isso ocorre por dois motivos, o primeiro é o fato do norte brasileiro ser uma região onde ocorrem grandes atividades convectivas, e o segundo ocorre pelo fato da região norte possuir apenas 8 estações para a geração dos dados do INMET/CPTEC, enquanto os dados do CHIRPS se referem às médias dos pixels de sua resolução espacial. 

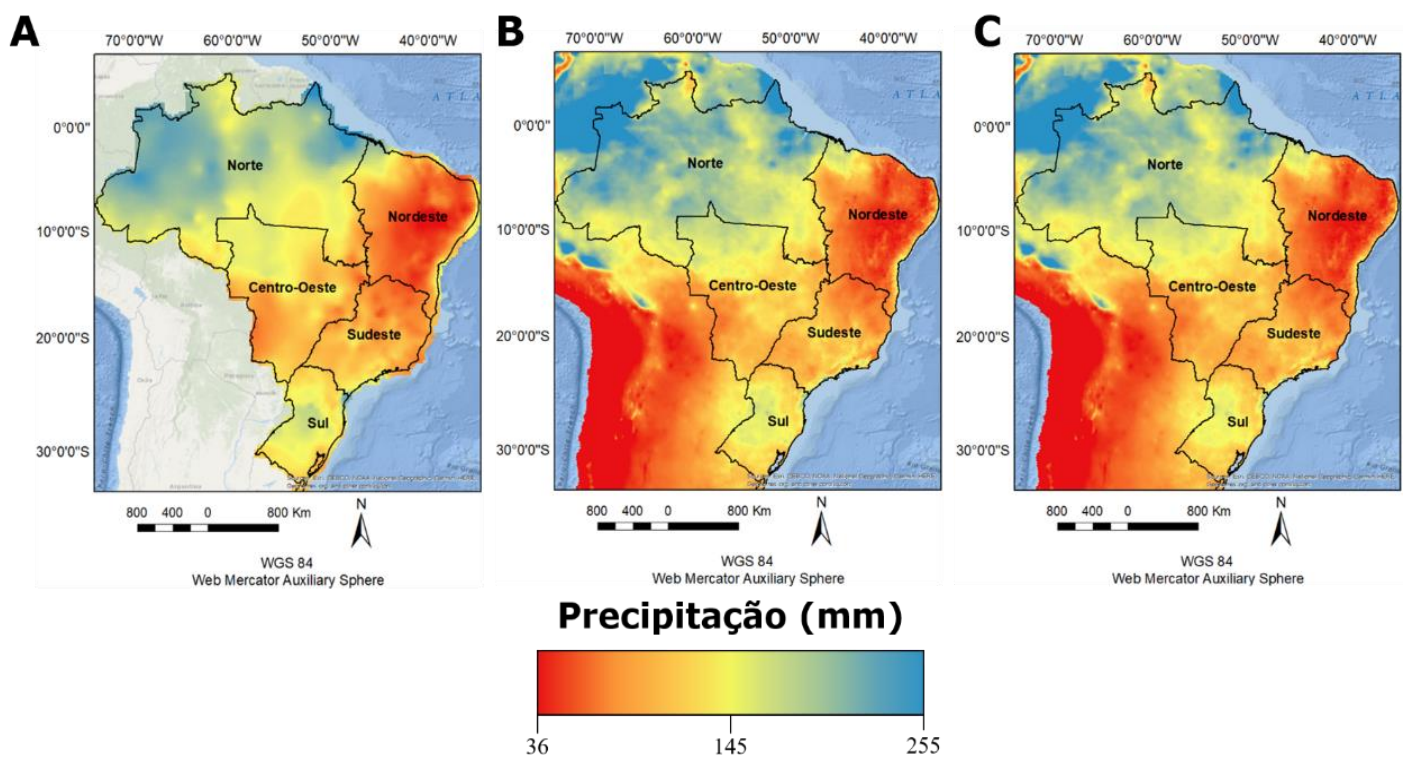

Figura 5 - Índices mensais de precipitação $(\mathrm{mm})$ para janeiro de 1998 a dezembro de 2010. (A) Distribuição espacial dos índices médios da precipitação anual estimada a partir dos dados INMET/CPTEC; (B) Distribuição espacial dos índices médios da precipitação anual estimada a partir do CHIRPS; (C) Índices médios da precipitação anual ajustados pelas regressões regionais aplicado aos dados do CHIRPS.

Por fim, com base nos resultados de superestimativa de cada região, foi originado um mapa (Figura $5 \mathrm{C}$ ) cujos valores médios da precipitação anual foram calibrados pelas regressões regionais, aplicados aos dados do CHIRPS. Dessa maneira, foi possível uma significativa melhora para a comparação entre os dados espaciais de todas regiões do Brasil. Para a região do Noroeste do Amazonas, os dados se aproximaram com os observados pelas estações (entre 140 a 200 mm). Já para região sudoeste do Pará, os resultados mostraram redução em relação aos dados anteriores, embora a espacialidade das informações continue similares, indicando ótima aproximação entre os dados (variando entre 145 a $215 \mathrm{~mm}$ ).

\section{CONSIDERAÇÕES FINAIS}

Em suma, foi possível analisar e comparar a similaridade das informações entre o conjunto de dados CHIRPS e as 183 estações meteorológicas espalhadas pelas regiões brasileiras, obtidas pelo INMET/CPTEC. Neste contexto, as informações do INMET/CPTEC e CHIRPS para as regiões do Brasil apresentaram um coeficiente de determinação semelhante correspondendo a $93 \%$ para o Centro-Oeste, $91 \%$ para o Sul, $93 \%$ para o Norte, $98 \%$ para o Sudeste, $97 \%$ para o Nordeste e $97 \%$ para todo o Brasil.

Ao analisar a espacialização dos dados, o resultado apresentou pouca heterogeneidade para determinadas regiões, destacando o noroeste da Amazônia e o sudoeste do Pará. Desse modo, foi realizado a calibração entre os dados do CHIRPS e INMET/CPTEC de acordo com os resultados da regressão linear, contribuindo desta maneira para o melhor entendimento da distribuição das informações. Os dados do INMET/CPTEC a partir de 1961 apresentam grandes erros e falhas, limitando desta forma uma validação mais consistente, 
sugerindo para futuros trabalhos uma comparação com outras fontes de dados. No geral, considerando os resultados de determinação, erros e distribuições espaciais, foi definida a validação dos dados do CHIRPS para o Brasil no período de 1998 a 2010 como dados confiáveis, portando plausíveis de utilização em distintas finalidades relacionadas às pesquisas climáticas.

\section{AGRADECIMENTOS} apoio.

Á Fundação de Amparo à Pesquisa de Minas Gerais (FAPEMIG) pelo

\section{REFERÊNCIAS BIBLIOGRÁFICAS}

AB'SABER, A. N. Espaços ocupados pela expansão dos climas secos na América do Sul, por ocasião dos períodos glaciais quaternários. Universidade de São Paulo/Instituto de Geografia, 1977.

ARAGÃO, L. E. O. C.; POULTER, B.; BARLOW, J. B.; ANDERSON, L. O.; MALHI, Y.; SAATCHI, S; PHILIPS, O. L.; GLOOR, E. Environmental change and the carbon balance of Amazonian forests. Biological Reviews, v. 89, n. 4, p. 913931, 2014.

BAYISSA, Y.; TADESSE, T.; DEMISSE, G.; SHIFERAW, A. Evaluation of SatelliteBased Rainfall Estimates and Application to Monitor Meteorological Drought for the Upper Blue Nile Basin, Ethiopia. Remote Sensing, v. 9, n. 7, p. 669, 2017.

CAMPAROTTO, L. B. et al. Validação de dados termopluviométricos obtidos via sensoriamento remoto para o Estado de São Paulo. Revista Brasileira de Engenharia Ambiental. v. 17, n. 6, p. 665-671, 2013. (a)

CAMPAROTTO, L. B. et al. Validation of temperature and rainfall data obtained by remote sensing for the state of São Paulo, Brazil. Revista Brasileira de Engenharia Agrícola e Ambiental, v. 17, n. 6, p. 665-671, 2013. (b)

CAVALCANTE, I. F. A.; FERREIRA, N. J.; SILVA, M. G. A. J. S.; DIAS, M. A. F. S. Tempo e clima no Brasil. São Paulo: Oficina de Textos, 2009. 463 p.

CERA, J. C.; FERRAZ, S. E. T. Variações climáticas na precipitação no sul do Brasil no clima presente e futuro. Revista Brasileira de Meteorologia, v. 30, n. 1, P.81-88, 2015.

COSTA, H. C.; MARCUZZO, F. F. N.; FERREIRA, O. M.; ANDRADE, L. R.; Espacialização e Sazonalidade da Precipitação Pluviométrica do Estado de Goiás e Distrito Federal. Revista Brasileira de Geografia Física. Recife, v.5, n.1, p.8710, 2012.

COMPO, G. P.; WHITAKER, J. S.; SARDESHMUKH, P. D. Feasibility of a 100year reanalysis using only surface pressure data. Bulletin of the American Meteorological Society, v. 87, n. 2, p. 175, 2006.

DEMBÉLÉ, M.; ZWART, S. J. Evaluation and comparison of satellite-based rainfall products in Burkina Faso, West Africa. International Journal of Remote Sensing, v. 37, b. 17, p. 3995-4014, 2016.

DUAN, Z., LIU, J.; TUO, Y.; CHIOGNA, G.; \& DISSE, M. Evaluation of eight high spatial resolution gridded precipitation products in Adige Basin (Italy) at multiple 
temporal and spatial scales. Science of The Total Environment, v. 573, p. 15361553, 2016.

ERAZO, B.; BOURREL, L.; FRAPPART, F.; CHIMBORAZO, O.; LABAT, D., DOMINGUEZ-GRANDA, L.; MATAMOROS, D.; MEJIA, R. Validation of Satellite Estimates (Tropical Rainfall Measuring Mission, TRMM) for Rainfall Variability over the Pacific Slope and Coast of Ecuador. Water, v. 10, n. 2, p. 213, 2018.

FIRPO, M. Â. F. Influências remotas das TSM dos Oceanos Pacífico e Atlântico e da oscilação Antártica na variabilidade climática interanual no Rio Grande do Sul e suas inter-relações. 2012. 234 p. Tese (Doutorado em Meteorologia) Instituto Nacional de Pesquisas Espaciais (INPE), São José dos Campos, 2012.

FEARNSIDE, P. M. Brazil's Amazonian forest carbon: the key to Southern Amazonia's significance for global climate. Regional Environmental Change, v. 18, n. 1, p. 47-61, 2018.

FUNK, C. PETERSON, P.; LANDSFELD, M.; PEDREROS, D.; VERDIN, J.; SHUKLA, S; HUSAK, G.; ROWLAND, J.; HARRISON, L.; HOELL, A. ; MICHAELSEN, J. The climate hazards infrared precipitation with stations-a new environmental record for monitoring extremes. Scientific data, v. 2, p. 150066, 2015.

HARADA, Y.; KAMAHORI, H.; KOBAYASHI, C.; ENDO, H.; KOBAYASHI, S.; OTA, Y.; TAKAHASHI, K. The JRA-55 Reanalysis: Representation of atmospheric circulation and climate variability. Journal of the Meteorological Society of Japan. Ser. II, v. 94, n. 3, p. 269-302, 2016.

MELLO, C. R.; SILVA, A. M. Modelagem estatística da precipitação mensal e anual e no período seco para o estado de Minas Gerais. Revista Brasileira de Engenharia Agrícola e Ambiental, v. 13, n. 1, p. 68-74, 2009.

MENEZES, J. A.; CONFAlONIERI, U.; MADUREIRA, A. P.; BRITO DUVAL, I.; SANTOS, R. B.; MARGONARI, C. Mapping human vulnerability to climate change in the Brazilian Amazon: The construction of a municipal vulnerability index. PloS one, v. 13, n. 2, p. e0190808, 2018.

NOGUEIRA, S. M. C.; MOREIRA, M. A.; VOLPATO, M. M. L. Evaluating Precipitation Estimates from Eta, TRMM and CHRIPS Data in the SouthSoutheast Region of Minas Gerais State-Brazil. Remote Sensing, v. 10, n. 2, p. 313, 2018.

PEREIRA, G. SILVA, M. E. S.; MORAES, E. C.; CARDOZO, F. S. Avaliação dos dados de precipitação estimados pelo satélite TRMM para o Brasil. Revista Brasileira de Recursos Hídricos, v. 18, N. 3, p. 139-148, 2013.

REBOITA, M. S.; GAN, M. A.; ROCHA, R. P.; AMBRIZZI, T. Regimes de precipitação na América do Sul: uma revisão bibliográfica. Revista Brasileira de Meteorologia. v. 2 5, n. 2, 2010.

SMITH, T. M. ARKIN, P. A.; REN, L.; SHEN, S. S. Improved reconstruction of global precipitation since 1900. Journal of Atmospheric and Oceanic Technology, v. 29 , n. 10, p. 1505-1517, 2012.

SOARES, A. S. D.; PAZ, A. R; PICCILI, D. G. A.; Avaliação das estimativas de chuva do satélite TRMM no Estado da Paraíba. Revista Brasileira de Recursos Hídricos, v. 21, n.2 p. 139-148, 2016. 
SWANN, A. L. S. LONGO, M.; KNOX, R. G.; LEE, E.; MOORCROFT, P. Future deforestation in the Amazon and consequences for South American climate. Agricultural and Forest Meteorology, v. 214, p. 12-24, 2015.

TOTÉ, C.; PATRICIO, D.; BOOGAARD, H.; VAN DER WIJNGAART, R.; TARNAVSKY, E.; FUNK, C. (2015). Evaluation of satellite rainfall estimates for drought and flood monitoring in Mozambique. Remote Sensing, v. 7, n. 2, p. 1758-1776, 2015.

TWARDOSZ, R.; CEBULSKA, M.; WALANUS, A. Anomalously heavy monthly and seasonal precipitation in the Polish Carpathian Mountains and their foreland during the years 1881-2010. Theoretical and Applied Climatology, p. 1-15, 2015.

VÁSQUEZ, P. L. M. N.; MOLION, L. C. B.; ARAUJO ABDALAD, M.; MOREIRA, D. M.; SANCHEZ, A. Historical analysis of interannual rainfall variability and trends in southeastern Brazil based on observational and remotely sensed data. CLIMATE DYNAMICS, v. 50, n. 3, p. 801-824, 2018.

WANDERLEY, H. S. SEDIYAMA, G. C.; JUSTINO, F. B.; ALENCAR, L. P. D.; DELGADO, R. C. Precipitation variability in the 'Sertão' of San Francisco in the State of Alagoas. Revista Brasileira de Engenharia Agrícola e Ambiental, v. 17, n. 7, p. 790-795, 2013.

ZAMBRANO, F.; WARDLOW, B.; TADESSE, T.; LILLO-SAAVEDRA, M.; LAGOS, O. Evaluating satellite-derived long-term historical precipitation datasets for drought monitoring in Chile. Atmospheric Research, v. 186, p. 26-42, 2017. 